\title{
Imaging in anorectal malformations: What does the surgeon need to know?
}

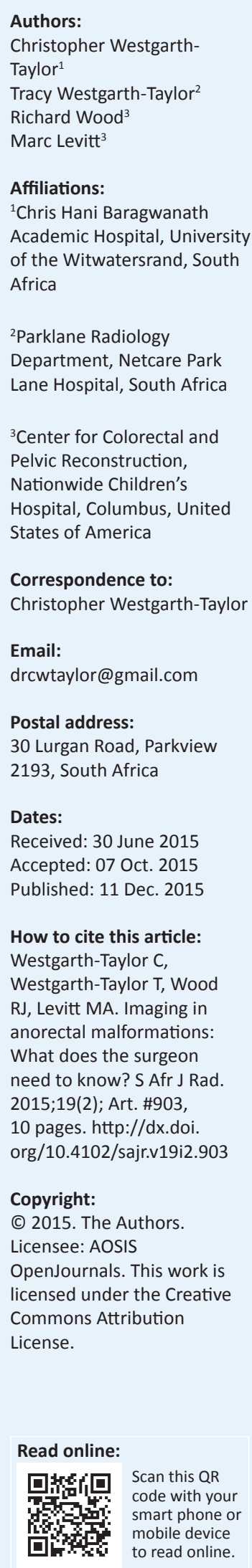

Imaging is essential in the diagnosis, management, surgical planning and eventual outcome in patients with anorectal malformation (ARM). This article outlines the imaging that may be required and the information needed by the surgeon to attain the best possible surgical outcome. ARM encompasses a wide spectrum of congenital malformations relating to the distal rectum and anus as well as the urinary and or gynaecological systems. The malformations range from a relatively simple perineal fistula with the potential for an excellent functional outcome, to complex cloacal malformation that requires specialist care and intervention. Approximately half of these children will have associated abnormalies. In the first $24-48$ hours of life, imaging is used to determine if any associated anomalies are severe enough to preclude an operation, and what operation will be required so that the child can grow as normally as possible. If a colostomy is done, pre-definitive repair imaging in the form of a high-pressure distal colostogram determines the surgical approach required to repair the malformation. In more complicated cases of cloacal malformation, advanced imaging in the form of MRI or 3D fluoroscopy is valuable. In the South African setting, 2D fluoroscopy with the surgeon present is adequate to help in planning for the surgical management. Communication between the radiologist and paediatric surgeon is essential to ensure that such patients have the best possible outcome.

\section{Introduction}

Anorectal malformation (ARM) encompasses a wide spectrum of congenital malformations relating to the distal rectum and anus as well as the urinary and or gynaecological systems. The malformations range from a relatively simple perineal fistula with the potential for an excellent functional outcome and a good anatomical reconstruction, to the complex cloacal malformation that requires specialist care and intervention. ${ }^{1}$ Approximately half of the children so affected will have an associated abnormality. The reported incidence is variable, but genitourinary $(40 \%-50 \%)$ is the most common, followed by cardiovascular $(30 \%-35 \%)$, spinal cord $(25 \%-30 \%)$, gastrointestinal (5\%-10\%), and vertebral defects, anal atresia, cardiac defects, tracheo-oesophageal fistula, renal anomalies and limb abnormalities (VACTERL) (4\%-9\%). ${ }^{2}$ ARM has a worldwide prevalence of approximately 1 in 5000 live births but, in the greater Gauteng area, the prevalence is 1 in 4000 live births. $^{3}$

\section{Classification of ARM}

The Krickenbeck classification (2005) is descriptive and places emphasis on the preoperative identification and anatomic evaluation of the rectal pouch and fistula. This information is vital for the surgeon as it allows anticipation of the extent of mobilisation in relation to the urinary tract as well as the most appropriate surgical approach for each case ${ }^{4}$ (Table 1).

\section{Management}

The aim of surgical intervention is to reconstruct the rectum/anus, genital tract and urinary tract. From a functional point of view, the goal is to be clean for stool and dry to urine. Many of these patients may require several medical or surgical interventions. Normal sexual function and the potential to bear children later in life are also very important long-term outcomes. ${ }^{5}$ Additionally, the urinary tract needs to be functional in such a way as to prevent long-term renal damage.

The early management of a newborn infant born with an ARM is crucial, and two questions must be answered:

1. Are there life-threatening associated abnormalities?

2. Is the child eligible for a primary procedure with no protective stoma, or is a protective decompressing stoma with definitive repair in 3-6 months required? 
TABLE 1: Krickenbeck classification of ARMs.

\begin{tabular}{ll}
\hline Major clinical groups & Rare/regional variants \\
\hline Perineal (cutaneous) fistula & Pouch colon \\
Rectourethral fistula & Rectal atresia/stenosis \\
Bulbar fistula & Rectovaginal fistula \\
Prostatic fistula & H-type fistula \\
Rectovesical fistula & Others \\
Vestibular fistula & - \\
Cloaca & - \\
ARM with no fistula & - \\
Anal stenosis & - \\
\hline
\end{tabular}

The decision is made on the basis of clinical features as well as radiological findings. Patients with a perineal/vestibular fistula will be eligible for a primary posterior sagittal anorectal plasty (PSARP); all other cases will require a defunctioning colostomy. Exceptions may have to be made if the baby's presentation is delayed and there is severe abdominal distension. In such cases, it is safer to decompress with a stoma than attempt a primary PSARP. The decision as to which management pathway to follow can only be taken after 24-48 hours of life. This delay allows time for gas to descend to the rectum and cause enough distension to establish if there is a fistula or not. During this time, it is essential to collate all possible radiological information to facilitate the decision process. See Figures 1 and 2 for the algorithm for neonatal management of male and female ARM patients respectively. Unfortunately, in the developing world, a significant number of these cases are missed at birth and they require a diverting colostomy regardless of the type of malformation, and therefore the algorithms do not apply in such cases.

\section{Imaging approach}

Imaging of patients with ARM can be divided into separate entities:

- antenatal diagnosis

- newborn evaluation

- high-pressure distal colostogram

- cloaca imaging

- follow-up imaging

- evaluation of complications.

\section{Antenatal}

The prenatal diagnosis of ARM on ultrasound (US) is rarely made. There have been reports that it has been diagnosed at 12 weeks' gestation, but the classical teaching is that it can be diagnosed at 22 weeks' gestation on the screening ultrasound (US). The difficulty in diagnosis probably results from the changing physiological characteristics of the fetal gastrointestinal tract, in addition to the small size of fetal structures in early pregnancy. ${ }^{6}$ The reported antenatal diagnosis rate is $16 \%-21 \% .{ }^{4,7}$ It is suggested that if anomalies associated with ARMs are identified on the scan, the examiner should intensify the search for an ARM. ${ }^{7}$ Oligohydramnios is present in $26 \%$ of ARMs. Other common prenatal imaging findings include an abdominal or pelvic cystic mass (52\%), fetal hydronephrosis (49\%), fetal ascites (22\%) and intestinal distension (18\%). If a distended bladder is identified with the diagnosis of oligohydramnios, the diagnosis of ARM should be considered. Cloacal malformations should be suspected if several pelvic cystic structures are identified on a scan, ${ }^{4}$ in a female fetus. Other associations include single kidney, absent radius and absent sacrum.

If there are features of a possible ARM on antenatal ultrasound, a fetal MRI in the third trimester should be considered. Urine normally has a signal intensity similar to that of simple fluid, with homogeneous hyperintensity on T2-weighted images and hypointensity on T1-weighted images, whereas normal meconium in the distal colon and rectum appears hyperintense on T1-weighted images and hypointense on T2-weighted images. In some fetuses with ARM (mainly recto-urethral fistulas in the male and long common-channel cloacal anomalies in the female), there is mixing of urine and meconium, thus resulting in heterogenous T1 and T2 signal intensity in the bladder and rectum. ${ }^{4}$ Having an antenatal diagnosis of an ARM allows better parental counselling and can also guide the site of delivery, where appropriate surgical facilities are available ${ }^{8}$ (Figure 3).

\section{Newborn evaluation}

The purpose of imaging in the first $24-48$ hours is to aid the surgeon's clinical examination and to decide which baby needs a decompressing colostomy and which baby would benefit from a primary definitive procedure without a covering stoma. At the same time, it is important to diagnose any associated anomalies that may influence the surgical decision-making in order to prevent potential unnecessary morbidity or mortality. Imaging required in the first $24-48$ hours includes: babygram (thorax, spine, limbs and pelvis), abdominal US, echocardiogram and spinal US. Additional imaging, but with much lower yield, may include a perineal US or a prone cross table lateral X-ray.

\section{Babygram}

A babygram should include the thorax, spine and pelvis in an AP and lateral projection. These images should allow identification of cardiac, costal, spinal and sacral abnormalities (Figure 4). If any sacral or vertebral anomalies are identified, this may be an indication for an MR spine in the future. The AP and lateral sacral views can identify anomalies such as a hemisacrum, sacral hemivertebrae, scimitar sacrum and sacral agenesis, and can also be used to identify the sacral ratio by measuring the distance between key bony structures. This is an important ratio that may help to predict future continence of the child (Figure 5).

A sacral ratio of 0.77 is accepted to be normal. It would be very unusual to have a sacral ratio of less than 0.3 and obtain adequate bowel control. ${ }^{9}$ If the limbs are included in the X-ray series, limb abnormalities such as radial aplasia may also be noted.

All this information assists in giving parents correct expectations regarding their child's functional outcome. 
Male anorectal malformation

VACTERL screening:

- Spinal radiograph

- Sacral radiographs

- Spinal US (<3 months old)

- MRI spine (>3 months old)

or if spinal USS abnormal

- ECHO

- NG tube and AXR to con rm

in stomach

- Renal US

- Limb radiographs

(as clinically indicated)

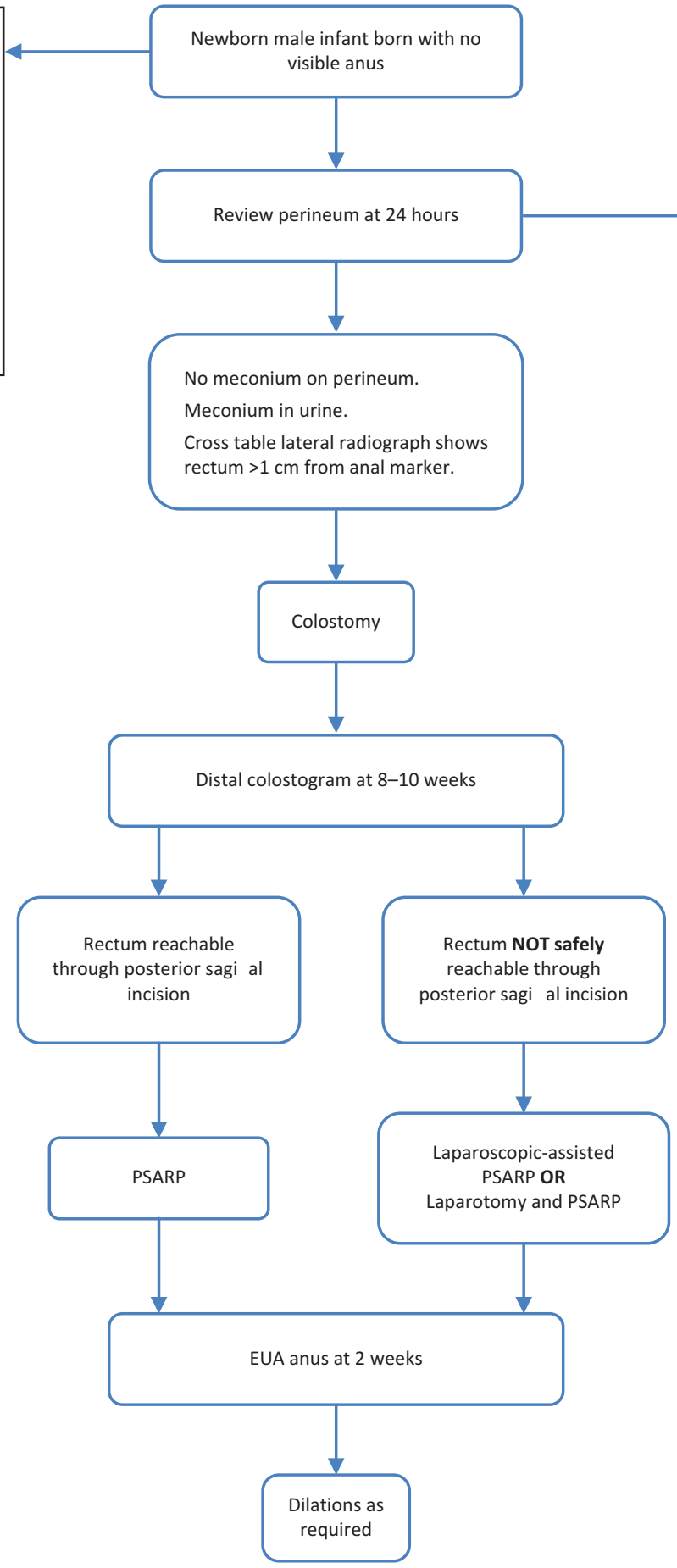

Meconium on perineum.

Cross table lateral radiograph shows rectum $\leq 1 \mathrm{~cm}$ from ana marker.

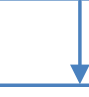

Primary PSARP

PSARP, posterior sagittal anorectoplasty; US, ultrasound scan; EUA, examination under anaesthetic; NG, nasogastric; RV, rectovaginal; CC, common channel

FIGURE 1: Male anorectal malformation.

\section{Ultrasound of the abdomen and pelvis}

Abdominal and pelvic US is used to detect any anomalies in the urogenital tract. Urological abnormalities are the most common abnormalities associated with ARMs. They are usually performed with an $8 \mathrm{MHz}$ transducer in both the prone and supine positions. Owing to the relative physiological oliguria that occurs in the first $24-48$ hours of life, imaging of the urinary tract can be misleading. ${ }^{4}$ It is important to repeat the US at 2 weeks if it was obtained in the first $24-48$ hours. In the case of a cloacal malformation, it is also important to identify a distended vagina (hydrocolpos), which will require surgical intervention to drain it. The hydrocolpos has two potential problems: (1) it can cause ureteric obstruction by compressing the trigone of the bladder, with resultant hydroureter and hydronephrosis, resulting 


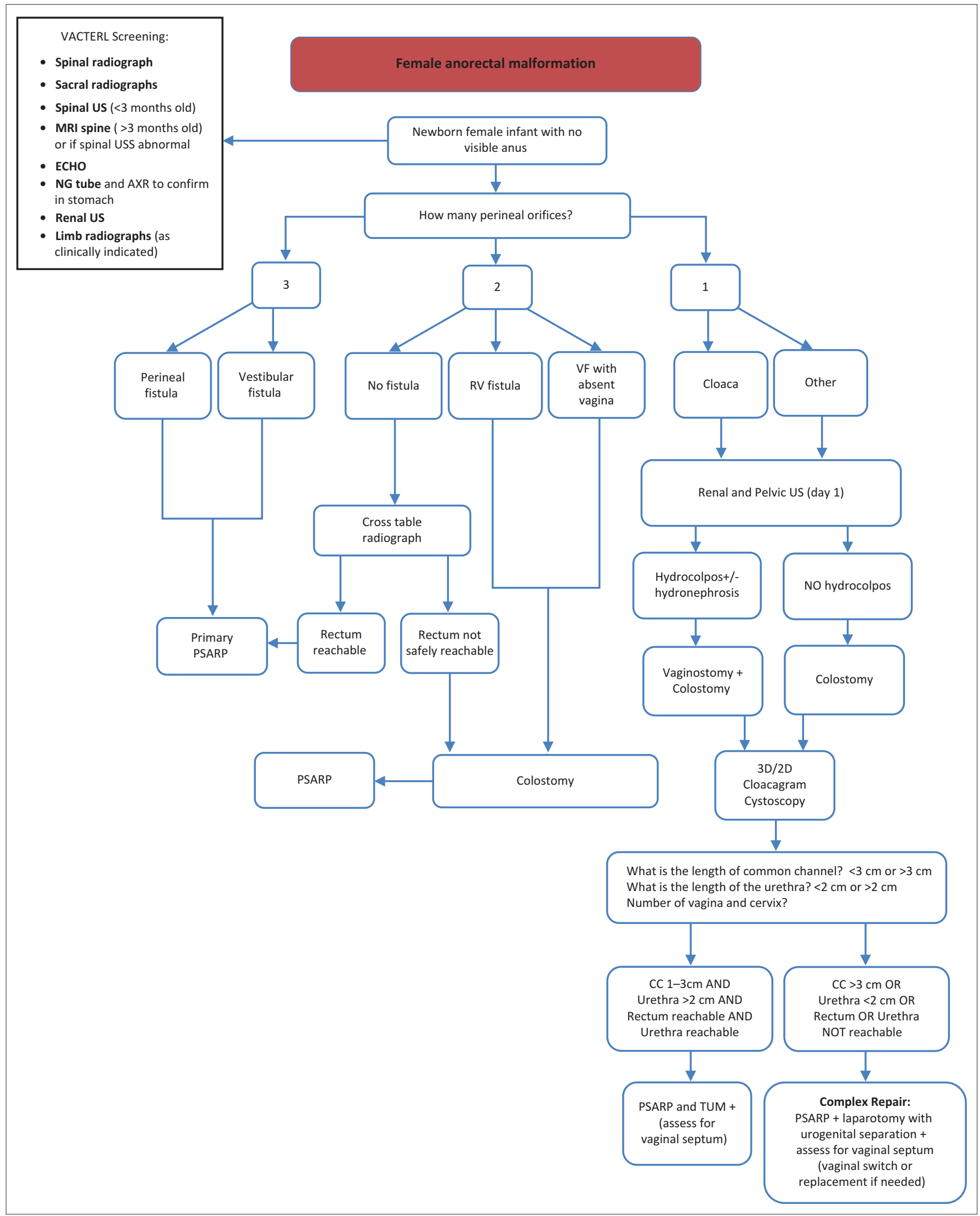

PSARP, posterior sagittal anorectoplasty; US, ultrasound scan; EUA, examination under anaesthetic; NG, nasogastric; RV, rectovaginal; CC, common channel; TUM, total urogenital mobilisation; $\mathrm{VF}$, vestibular fistula

FIGURE 2: Female anorectal malformation. 


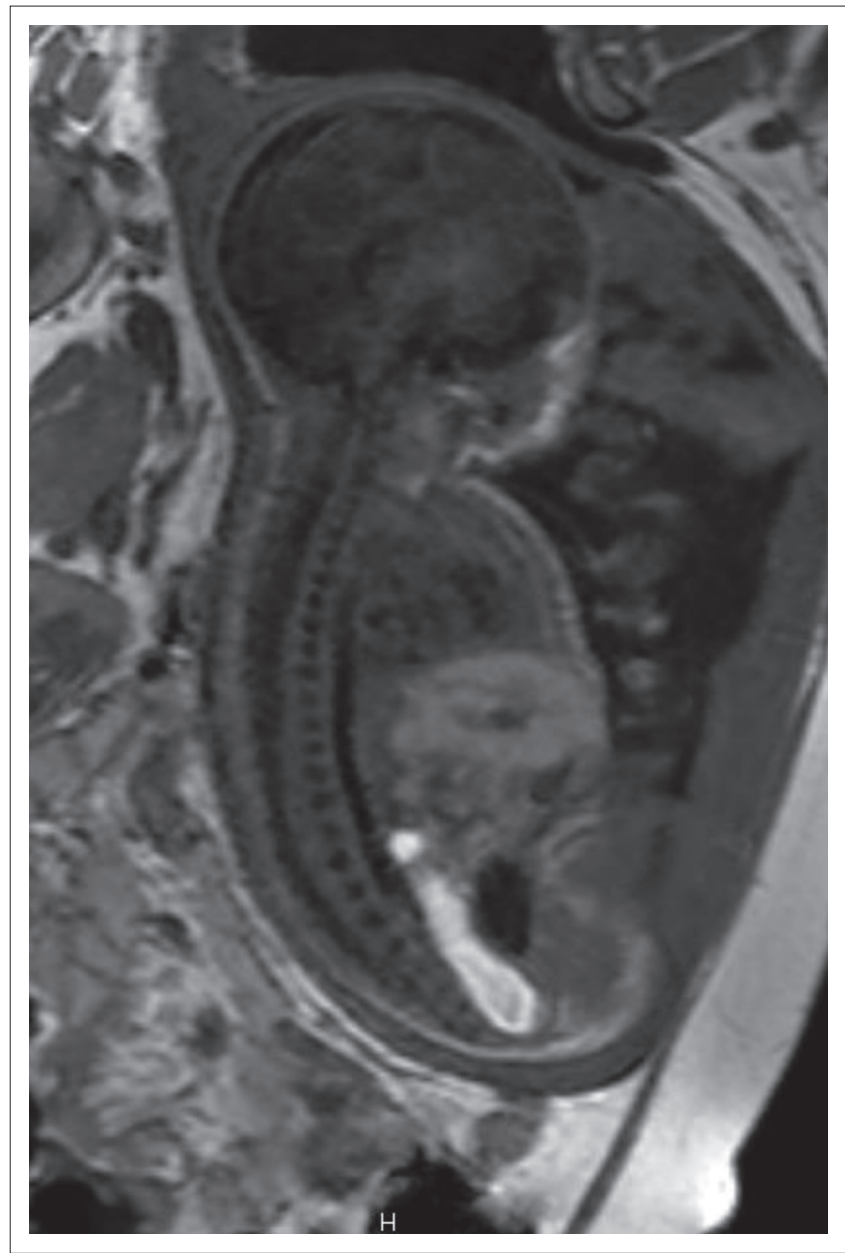

FIGURE 3: Midline sagittal T1W fetal MRI demonstrating a dilated rectum with some areas of T1W hypointensity in the central rectum representing mixing of meconium and urine.

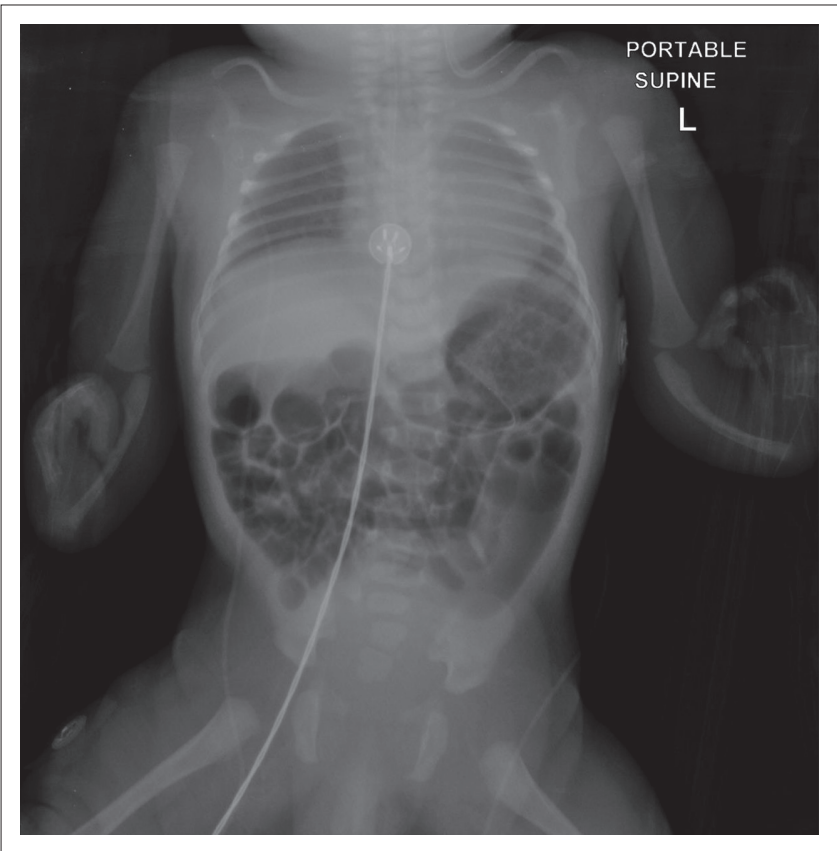

FIGURE 4: Supine radiograph demonstrating bilateral radial aplasia and sacra abnormalities.

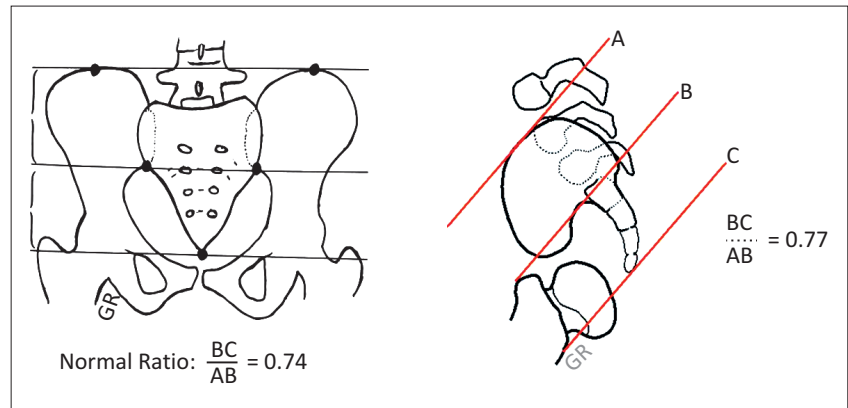

FIGURE 5: Schematic diagram illustrating how the sacral ratio is measured.

in obstructive uropathy; and (2) the second, much rarer, problem is that it can become infected and form a pyocolpos that can be potentially life threatening to a newborn baby if not urgently drained. ${ }^{5}$ Pre-sacral masses also need to be identified especially if there are sacral abnormalities as they may indicate a Currarino triad - an autosomal dominant condition consisting of an ARM, presacral mass and bony defect of the sacrum. These masses are usually teratomas, but can also be meningocoeles or dermoid cysts. Because of possible malignant transformation, these masses need to be removed at the time of surgery.

\section{Spinal ultrasound}

Controversy exists regarding the best form of spinal imaging in patients with ARMs. The teaching was that spinal US under the age of 3 months was a good screening tool to rule out spinal abnormalities such as spinal cord tethering, lipomas or spinal dysraphism. ${ }^{4}$ Recent studies are refuting this view, owing to the high number of false negatives obtained when comparing US to MRI. False negatives were found in $21 \%$ and $53 \%$ of cases when comparing ultrasound with MRI in studies by Kim et al. and Scottoni et al. respectively. ${ }^{10,11}$

There are obvious limitations to each modality; but US is safe, inexpensive, non-invasive, does not require sedation and can be performed at the bedside. MRI, on the other hand, is expensive, time consuming, may require sedation or anaesthetic, and is not available at all centres. It is probably safe to say that all children with an abnormality detected on US or X-ray require an MRI spine. Some centres do not perform spinal US but go straight to a spinal MRI at 6 months of age.

\section{Prone cross table lateral X-ray}

The cross table lateral $X$-ray can be used on rare occasions when the level of the fistula has not declared itself after 24 hours. ${ }^{12,13}$ The aim of the investigation is to try to determine if the lesion is 'low' and can therefore have a primary definitive procedure without a covering stoma. The position of the anus should be marked with a radio-opaque material, preferably barium paste or a ball bearing, and the distance from the skin to the rectal gas measured. If the distance is less than $1 \mathrm{~cm}$, the patient is probably a candidate for a primary procedure; if greater than $1 \mathrm{~cm}$, a decompressing stoma should be performed ${ }^{1}$ (Figure 6). An ARM patient without a fistula is extremely uncommon and is commonly associated with trisomy 21 . 


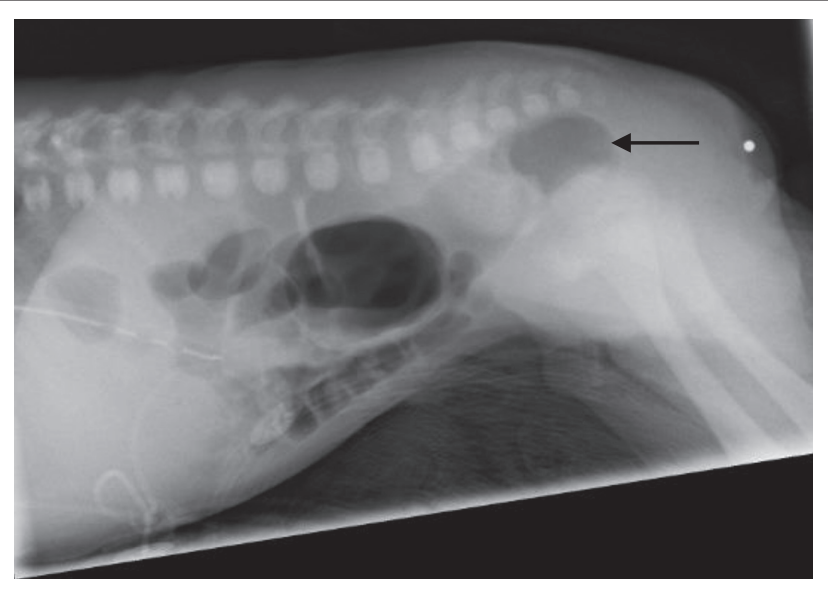

FIGURE 6: Prone cross table lateral radiograph of a neonate at 24 hours of age demonstrating a dilated rectum (arrow) measuring $>1 \mathrm{~cm}$ from the anal marker.

\section{Perineal ultrasound}

Perineal US has been used to measure the distance of the distal rectal pouch to the skin and to identify any recto-urogenital fistula. A 10-12 MHz high-frequency linear array transducer is normally used with the child in the supine position with pelvis and legs raised. A reference of $15 \mathrm{~mm}$ has been used to determine the difference between a 'high' and 'low' lesion. It must be cautioned that any straining or crying during the procedure can increase the intra-abdominal pressure and displace the rectal pouch into a falsely 'low' position. Another problem is the probe itself indenting the perineum and once again falsely reassuring the operator of a 'low' lesion. ${ }^{4}$ Many surgeons do not find this examination useful as it is very operator dependent and there are numerous factors involved that may alter the results.

\section{Micturating cystourethrogram (MCUG)/voiding cystourethrogram}

The MCUG is an important study to do if abnormalities have been detected on the abdominal ultrasound or spinal US/MRI. It enables the diagnosis of associated congenital urological problems, the most common being vesico-ureteric reflux (VUR). MCUG may also demonstrate the location of a recto-urinary fistula, but is not considered the best method of visualising the fistula. ${ }^{4}$

\section{Echocardiogram}

An echocardiogram is essential to obtain prior to any surgical intervention to rule out cardiovascular anomalies that could cause problems during anaesthesia. Cardiovascular anomalies are the second most common association occurring in ARM after urological anomalies, with the former occurring in about $30 \%-35 \%$ of patients.

\section{High-pressure distal colostogram}

The high-pressure distal colostogram is the most accurate way of determining the anatomy of the distal rectal pouch and fistula in boys. ${ }^{12,13}$ The investigation is ideally done at

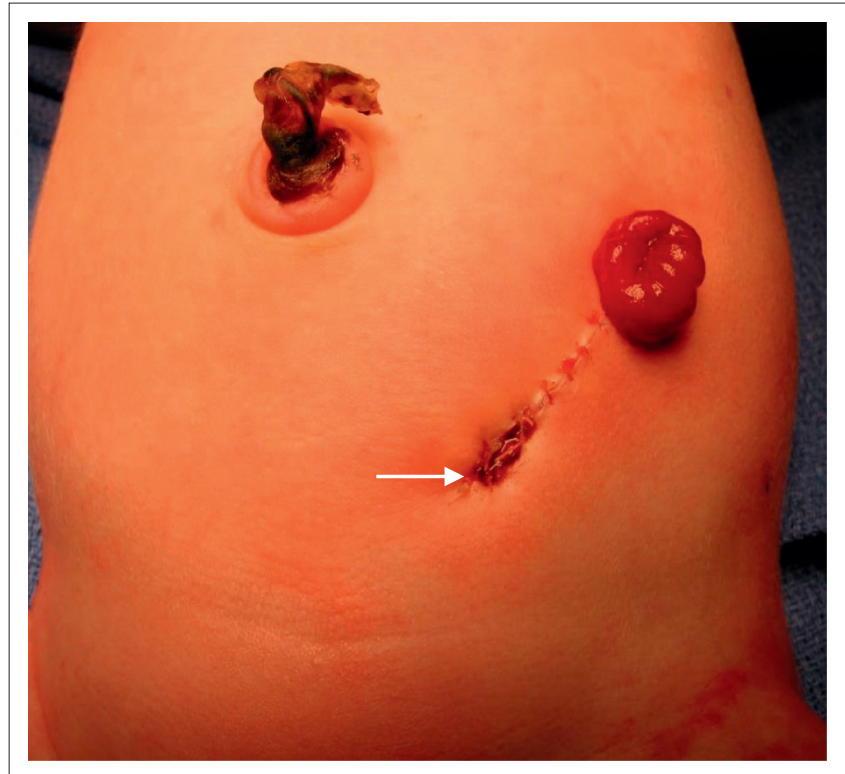

FIGURE 7: Immediate postoperative image of the anterior abdomen showing the location of the divided stoma; arrow indicates the distal loop, which would be catheterised at colostogram.

2-3 months of age in ARM patients who have undergone a diverting colostomy; it should be done just prior to the definitive repair. The most common stoma done in patients with ARM is a divided stoma with a skin bridge between the proximal and distal limb. The distal limb is made small and flush with the skin and will appear close to the midline in the lower abdomen (Figure 7). Sometimes the skin grows over the distal opening and it can appear quite small. The surgeon's assistance may be necessary to find the ostia.

A Foley catheter is then inserted into the distal fistula and $3 \mathrm{ml}$ of saline is injected into the balloon. Gentle traction is applied to the catheter to create a seal so that adequate pressure can be applied when injecting the contrast. A radioopaque marker is placed where the anus should be. Contrast medium (water soluble) is injected via the Foley catheter with a $50 \mathrm{ml}$ catheter tip syringe into the rectum under gentle but increasing pressure until the distal rectum and fistula are identified. The AP view is pivotal to see how much distal bowel is present, and the lateral view then defines the distal rectal anatomy as it relates to the urinary tract. Fluoroscopy with spot filming with the child in AP and then lateral position is performed. Sufficient pressure must be applied to distend the distal rectum adequately and identify the fistula.

It is worth noting that too much pressure may cause bowel perforation or decrease the actual distance from distal rectum to skin, giving the impression of a falsely low pouch. However, too little pressure will give the false impression of a high rectum with no fistula; this will show up as a flattening of the distal rectum at the pubococcygeal line, and means more pressure is required for an accurate study. In most cases where there is not a fistula to the bladder, the study will be considered completed once contrast has reached the level below the pubococcygeal line, indicating that enough pressure has been applied. The sacrum, coccyx, perineum 
and bladder must all be visible during fluoroscopy. If a fistula is identified, it is suggested to continue injecting the contrast until the bladder fills and the baby spontaneously voids. If this is the case, valuable information regarding the urinary tract can be obtained. It is in essence a modified $\mathrm{MCUG}^{14}$ (Figures 8-10).

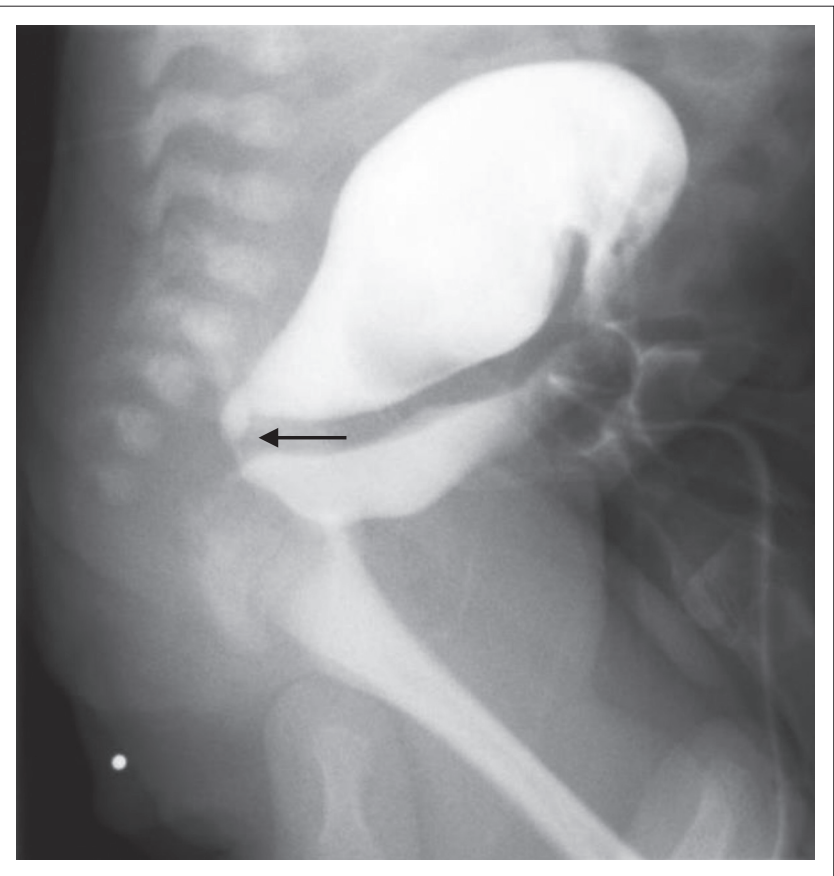

FIGURE 8: Lateral radiograph during high-pressure distal colostogram showing a recto-vesical fistula (arrow).

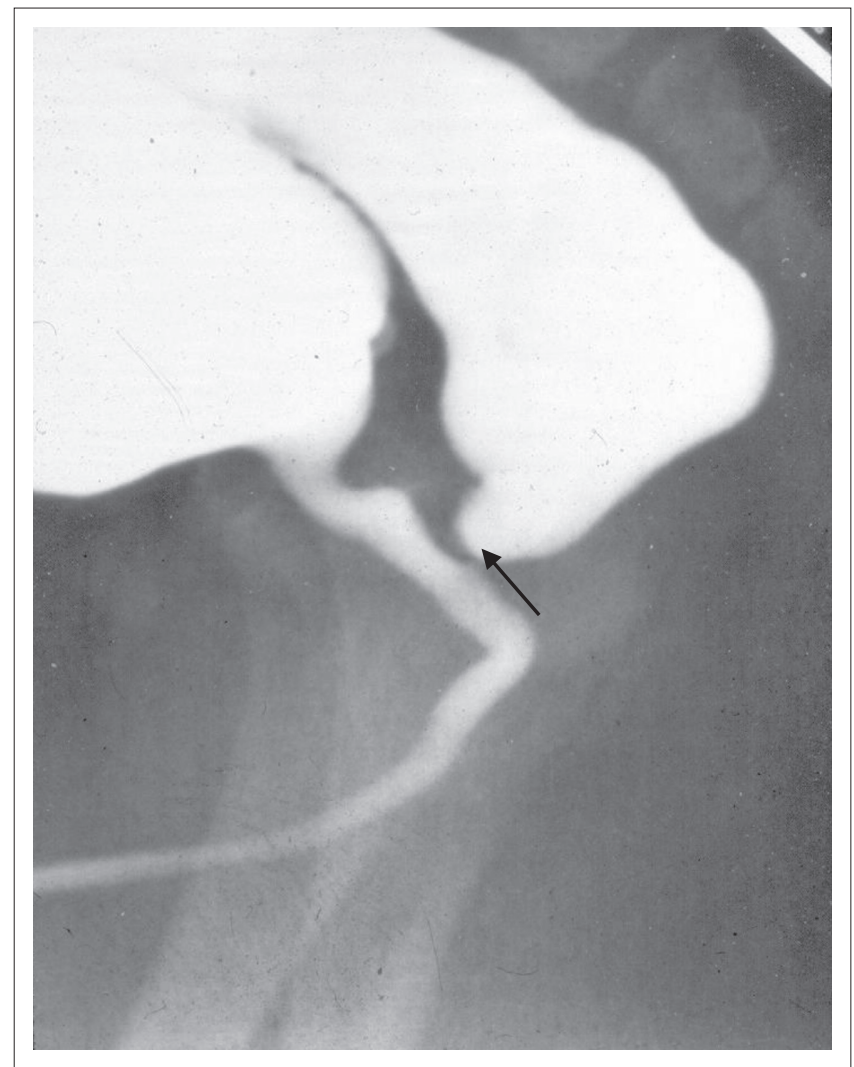

FIGURE 9: Lateral radiograph during high-pressure distal colostogram showing a recto-prostatic fistula (arrow).

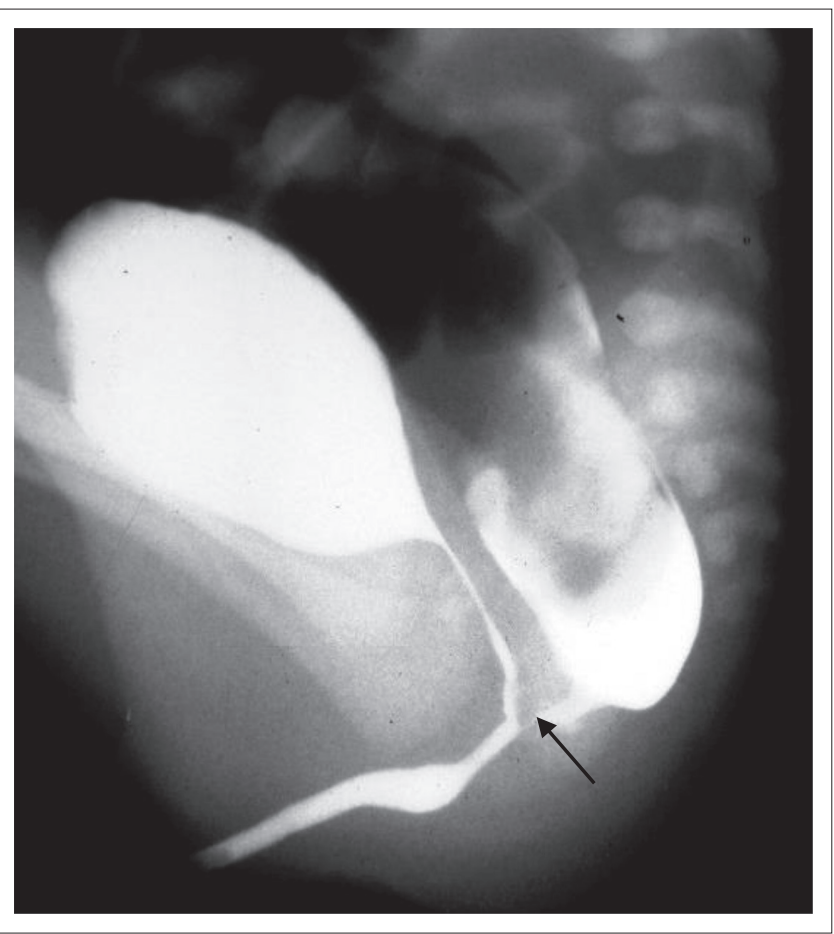

FIGURE 10: Lateral radiograph during high-pressure distal colostogram showing a recto-bulbar fistula (arrow).

The information that the surgeon would like to know is the precise location of the fistula in relation to the urinary tract, the length of bowel available for the pullthrough procedure, and the relationship between the sacrum, coccyx and rectum. All this information is vital to plan the type of operation required (PSARP, laparotomy or laparoscopy). ${ }^{9}$ The usual surgical approach for a bulbar or low prostatic fistula would be PSARP. For a vesical or high prostatic fistula, the approach would normally be laparotomy or laparoscopy for ligating the fistula and mobilising the bowel, then a limited PSARP incision to bring it down to the cutaneous junction in the centre of the muscle complex. It must be emphasised, however, that not every patient fits this formula, and one may find a low prostatic fistula that has a long fistula with a rectal stump that is high. Such a patient would do best with a laparotomy/laparoscopy first, followed by a limited PSARP (Figure 11).

In lieu of doing colostograms, many centres do pelvic MR imaging to determine the position of the fistula, and are obtaining good results. MRI avoids radiation, and increases detection of spinal dysraphism, tethered cord and pre-sacral masses; it can also show sphincter and pelvic floor musculature preoperatively. The disadvantages are that the procedure may require sedation/anaesthesia, is expensive and logistically demanding. ${ }^{15,16}$ Our view is that MRI is not yet able to replace the high-pressure distal colostogram for accurately demonstrating the anatomy required for preopertaive planning, but certainly plays a role in imaging patients who have previously been operated on. 


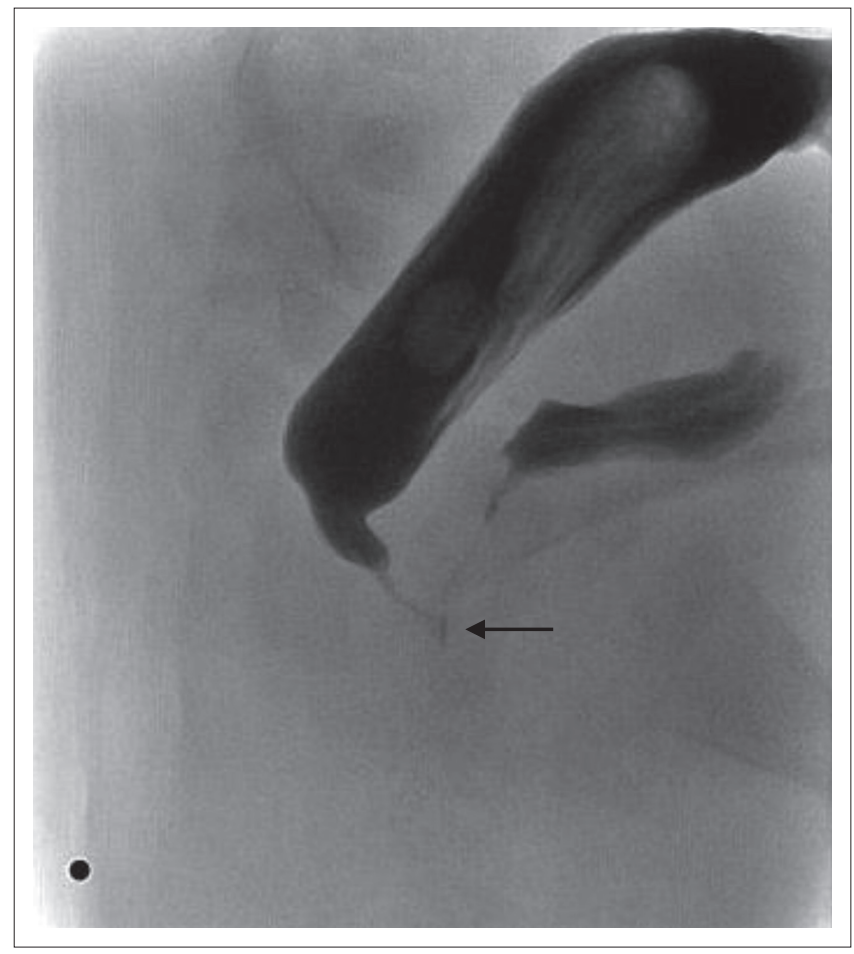

FIGURE 11: Lateral radiograph during high-pressure distal colostogram showing a recto-bulbar fistula with a high rectal pouch and long fistula (arrow).

\section{Cloacal malformation}

Cloacal malformation is the most difficult technical challenge in the spectrum of ARMs. The defect involves fusion of the urethra, vagina and rectum to form a common channel. Above the level of the common channel, the rectum and the vagina share a common wall, as do the urethra and vagina. The length of the common channel is vitally important to the surgeon, and a cut-off of a channel length of $3 \mathrm{~cm}$ is used to determine the surgical technique used to repair the malformation. With a channel length of less than $3 \mathrm{~cm}$, theoretically a posterior sagittal procedure involving PSARP and total urogenital mobilisation should suffice in the repair of these children. It is reproducible and can be done by most paediatric surgeons. With a common channel of greater than $3 \mathrm{~cm}$, a combined abdomino-perineal approach is recommended. The repair of the rectum is similar to any other ARM, but it is the repair of the vagina, urethra and associated urological problems that presents the main surgical challenge. Such cases should be sent to a specialist centre with paediatric surgeons who have a special interest in these conditions and perform the operations regularly. ${ }^{5}$

All cloacal malformations should receive a primary stoma to decompress them prior to the definitive repair at 3-6 months. As previously mentioned, it is imperative that all patients with cloacal malformations receive an urgent abdominal and pelvic US to rule out hydrocolpos and its associated complications. If a hydrocolpos is present, it can be drained at the time of the colostomy creation.

Before any definitive surgical management is attempted, it is important to identify the anatomy of the malformation

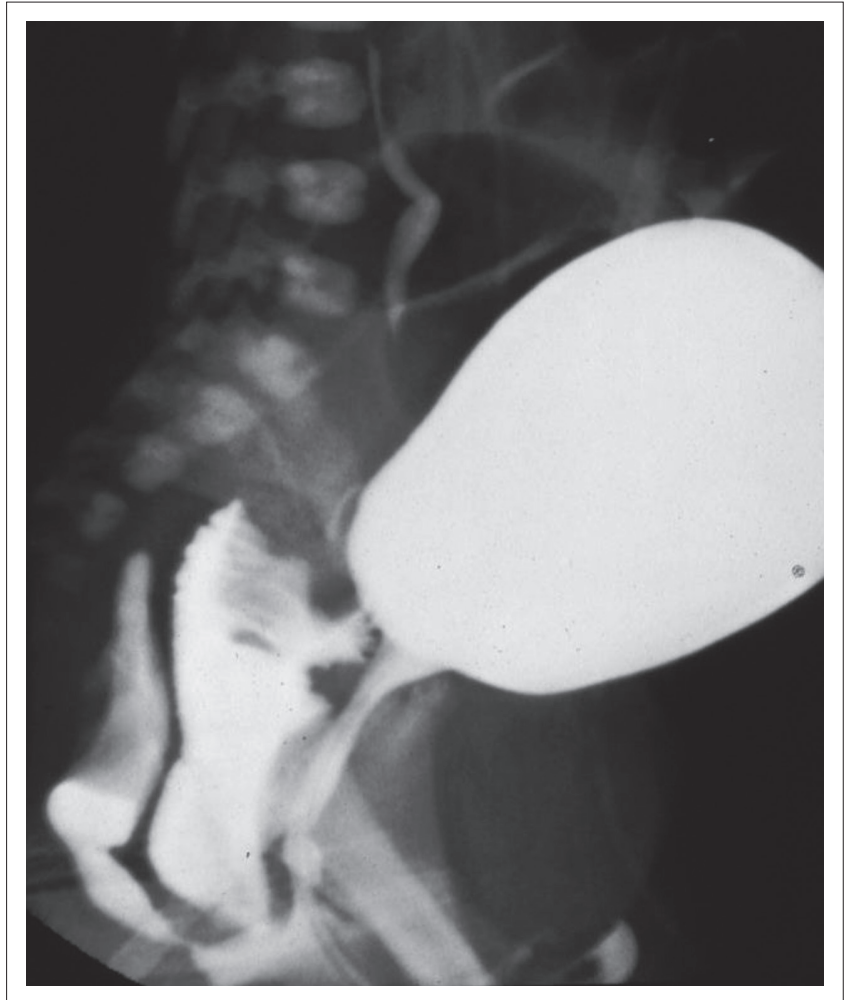

FIGURE 12: Lateral radiograph during cloacogram.

and its associated urogenital anomalies; this is done by endoscopy combined with a cloacagram. The endoscopy is done by the paediatric surgeon and involves cystoscopy and vaginoscopy. At this point, it is essential to measure the length of the common channel and insert catheters into the respective orifices so that a cloacagram can be performed in the radiology suite at a later time. A problem with 2D fluoroscopy is that it can be difficult to interpret overlapping structures. ${ }^{17}$ Since it is a dynamic study, it would be appropriate to have the surgeon in the room during the study. It must be remembered that approximately $40 \%$ of patients have a double Mullerian system consisting of 2 hemi-uteri and 2 hemi-vaginas; this can make interpretation very difficult. It can also be difficult to measure distance with 2D fluoroscopy, which is vital to know when planning a definitive repair (Figure 12).

Rotational fluoroscopy with 3D reconstruction is showing promise in negating the shortfalls of 2D fluoroscopy. Vaginal anatomy and anomalies can be identified using 3D reconstruction. Vaginal characteristics, such as location, size and presence of hemi-vaginas, are important to identify preoperatively in order to determine the best approach to the repair of the genital component of the malformation. ${ }^{17}$ Other key factors are common channel length, and length from urethra to bladder neck.

Because of magnification and foreshortening with conventional 2D screening, measuring the length of the common channel can be difficult. With rotational fluoroscopy, this is more accurate and the surgical approach can thus 
be determined accurately. ${ }^{17}$ The length of the distal colon and rectum can be more accurately measured, especially if there is redundancy in the loop, which can be missed in 2D fluoroscopy. This reassures the surgeon that there is sufficient length of distal rectum to pull through to the perineum.

Disadvantages of 3D fluoroscopy are that more radiation is required and a general anaesthetic is suggested to reduce movement artefact. The centres that use this technique have determined that the information obtained with this technique far outweighs the disadvantages because it supplies the surgeon with precise anatomical information to facilitate accurate surgical planning of a cloacal malformation and to define prognoses regarding long-term bowel, bladder and sexual function. ${ }^{17}$

Other centres have taken 3D fluoroscopy to the next step and added low-osmolality iodinated contrast material mixed with a small amount of gadolinium. Contrast material is used to distend the catheterised cavities and a high-resolution 3D MRI of the pelvis is performed, which allows soft-tissue structures outside the lumens of the vagina, rectum and bladder to be well defined. ${ }^{18}$

Not all centres have the luxury of being able to offer such advanced imaging techniques, but this can be overcome with a well-planned cloacagram (2D fluoroscopy), ideally with the operating surgeon in the radiology suite with the radiologist.

\section{Follow-up imaging}

After definitive repair of patients, it must be remembered that a large number might have associated renal abnormalities. The most common anatomical abnormalities observed are dysplastic kidneys or an absent kidney, followed by hydronephrosis. The most common functional defect is VUR. Surveillance of these anomalies is usually left to the discretion of the urologist and nephrologist. There is no doubt, however, that they will need follow-up by US, MCUG and nuclear medicine. In patients with ARM, their most significant morbidity is from their urological problems. ${ }^{9}$

\section{Imaging of complications}

Despite advances in surgical repair, complications are still common. These can be in the form of a persistent fistula, mislocated rectum, posterior urethral diverticulum, rectal stricture and a persistent urogenital sinus. MRI seems to be the appropriate imaging technique in these patients to determine if reoperative surgery is required and to plan the surgical technique $^{19}$ (Figures 13 and 14).

\section{Conclusion}

Imaging in patients with ARM is vital in determining the correct surgical management. In the first $24-48$ hours, imaging is used to determine if any associated anomalies are severe enough to preclude an operation, and what operation will be required. If a colostomy is done, the pre-definitive repair imaging in

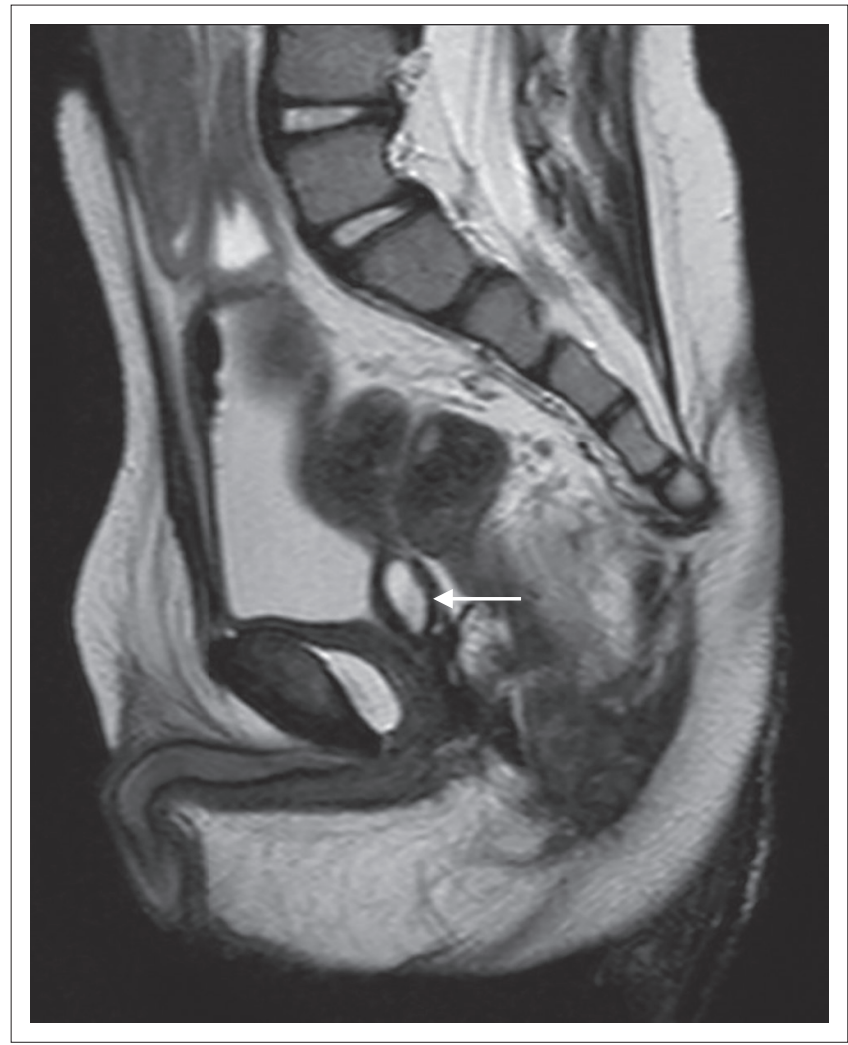

FIGURE 13: Sagittal T2W MRI of the pelvis showing a posterior urethra diverticulum (arrow).

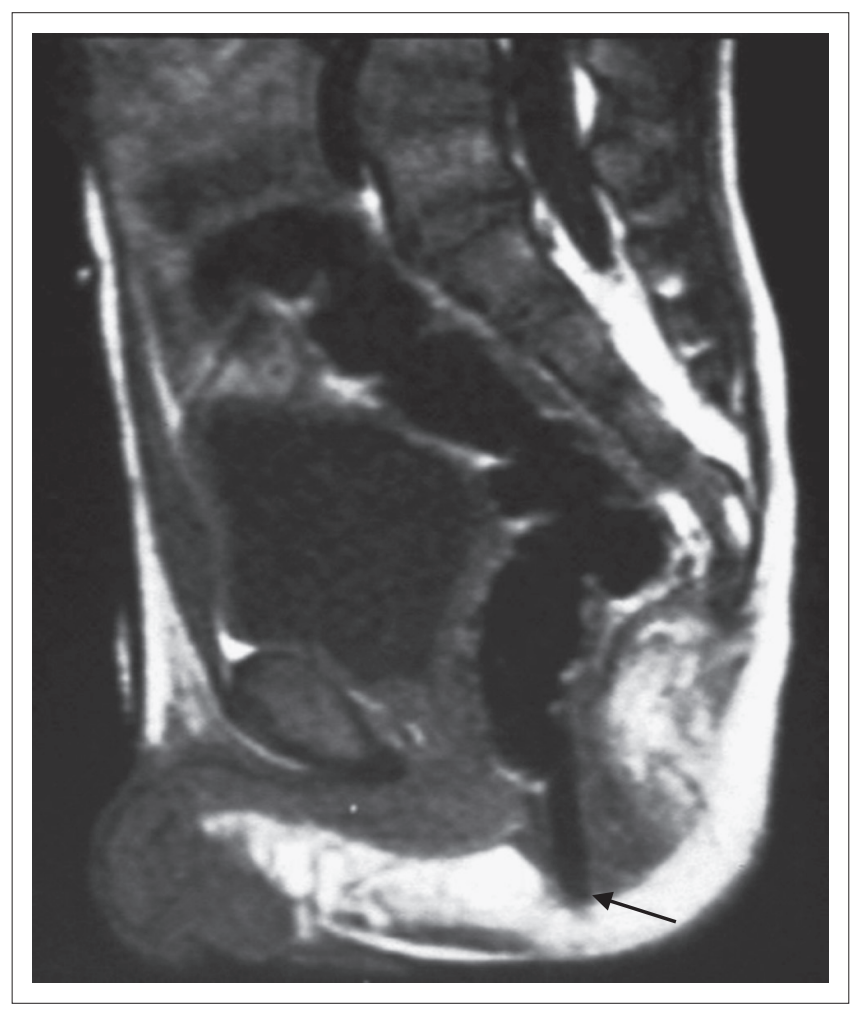

FIGURE 14: Sagittal T1W MRI of the pelvis showing the postoperative mislocated neo-anus anterior to the muscle complex (arrow).

the form of a high-pressure distal colostogram determines the surgical approach required to repair the malformation. In more complicated cases such as persistent cloaca, advanced imaging in the form of MRI or 3D fluoroscopy is valuable. 
Although these advanced imaging modalities may add important information in planning surgery for these patients, 2D fluoroscopy images with the surgeon present is in most settings the most important radiological investigation by far to help planning for the surgical management.

\section{Acknowledgements Competing interests}

The authors declare that they have no financial or personal relationships which may have inappropriately influenced them in writing this article.

\section{Authors' contributions}

C.W.T. (University of the Witwatersrand) wrote most of the manuscript. T.W.T. (Parklane Radiology), R.W. (Center for Colorectal and Pelvic Reconstruction) and M.L. (Center for Colorectal and Pelvic Reconstruction) contributed individually to the manuscript. Images were provided by RW, ML and TWT.

\section{References}

1. Levitt MA, Peña A. Anorectal malformations. Orphanet J Rare Dis. 2007;2:33. PMID 17651510, http://dx.doi.org/10.1186/1750-1172-2-33

2. Gangopadhyay A, Pandey V. Anorectal malformations. J Indian Assoc Pediatr Surg 2015;20:10. PMID: 25552824, http://dx.doi.org/10.4103/0971-9261.145438

3. Theron A, Loveland J. Birth prevalence of anorectal malformation in the referral area for the University of the Witwatersrand Tertiary Hospitals, South Africa. Eur J Pediatr Surg Off. 2015;25:220-225. http://dx.doi.org/10.1055/s-0033-1360456

4. Alamo L, Meyrat BJ, Meuwly J-Y, Meuli RA, Gudinchet F. Anorectal malformations: Finding the pathway out of the labyrinth. Radiographics. 2013;33:491-512. PMID: 23479709, http://dx.doi.org/10.1148/rg.332125046

5. Levitt MA, Peña A. Cloacal malformations: Lessons learned from 490 cases. Semin Pediatr Surg. 2010;19:128-138. http://dx.doi.org/10.1053/j. sempedsurg.2009.11.012
6. Gilbert CE, Hamill J, Metcalfe RF, Smith P, Teele RL. Changing antenatal sonographic appearance of anorectal atresia from first to third trimesters. J Ultrasound Med. 2006;25:781-784. PMID: 16731896.

7. Brantberg A, Blaas H-G, Eik-Nes SH. P14. 37: Anorectal obstructions: A rare prenatal but frequent postnatal diagnosis. Ultrasound Obstet Gynecol. 2004;24:357. http:// dx.doi.org/10.1002/uog.1637

8. Calvo-Garcia MA, Kline-Fath BM, Levitt MA, et al. Fetal MRI clues to diagnose cloacal malformations. Pediatr Radiol. 2011;41:1117-1128. PMID: 21409544, http://dx.doi. org/10.1007/s00247-011-2020-8

9. Bischoff $A$, Levitt MA, Peña A. Update on the management of anorectal malformations. Pediatr Surg Int. 2013;29:899-904. PMID: 23913263, http://dx.doi. org/10.1007/s00383-013-3355-z

10. Kim SM, Chang HK, Lee MJ, et al. Spinal dysraphism with anorectal malformation: Lumbosacral magnetic resonance imaging evaluation of 120 patients. J Pediatr Surg. 2010;45:769-776. http://dx.doi.org/10.1016/j.jpedsurg.2009.10.094

11. Scottoni F, lacobelli BD, Zaccara AM, Totonelli G, Schingo AMS, Bagolan P. Spinal ultrasound in patients with anorectal malformations: Is this the end of an era? Pediatr Surg Int. 2014;30:829-831. PMID: 24996639, http://dx.doi.org/10.1007/ s00383-014-3546-2

12. Levitt MA, Peña A. Imperforate anus and cloacal malformations. In: Holcomb GW III, Murphy JP, editors. Ashcraft's pediatric surgery. 5th ed. Philadelphia: Saunders Elsevier; 2010; p. 468-490.

13. Peña A, Bischoff A. Surgical treatment of colorectal problems in children. Berlin Springer; 2015.

14. Gross GW, Wolfson PJ, Pena A. Augmented-pressure colostogram in imperforate anus with fistula. Pediatr Radiol. 1991;21:560-562. PMID: 1815175, http://dx.doi. org/10.1007/BF02012597

15. Kavalcova L, Skaba R, Kyncl M, Rouskova B, Prochazka A. The diagnostic value of MRI fistulogram and MRI distal colostogram in patients with anorectal malformations. J Pediatr Surg. 2013;48:1806-1809. http://dx.doi.org/10.1016/j. jpedsurg.2013.06.006

16. Alves JCG, Sidler D, Lotz JW, Pitcher RD. Comparison of MR and fluoroscopic mucous fistulography in the pre-operative evaluation of infants with anorectal malformation: A pilot study. Pediatr Radiol. 2013;43:958-963. PMID: 23460374, http://dx.doi.org/10.1007/s00247-013-2653-x

17. Patel MN, Racadio JM, Levitt MA, Bischoff A, Racadio JM, Peña A. Complex cloacal malformations: Use of rotational fluoroscopy and 3-D reconstruction in diagnosis and surgical planning. Pediatr Radiol. 2012;42:355-363. PMID: 22072072, http:// dx.doi.org/10.1007/s00247-011-2282-1

18. Jarboe MD, Teitelbaum DH, Dillman JR. Combined 3D rotational fluoroscopic-MR cloacagram procedure defines luminal and extraluminal pelvic anatomy prior to

19. Podberesky DJ, Towbin AJ, Eltomey MA, Levitt MA. Magnetic resonance imaging of anorectal malformations. Magn Reson Imaging Clin N Am. 2013;21:791-812. http://dx.doi.org/10.1016/j.mric.2013.04.010 